\title{
Seroprevalence of Helicobacter pylori among Healthy Blood Donors
}

\author{
D. Ramya Priyadarshini ${ }^{1 *}$, Joshy M. Easow ${ }^{2}$ and R. Vinod ${ }^{1}$ \\ ${ }^{1}$ Department of Microbiology, Sri Venkateshwara Medical College Hospital and Research \\ Centre (SVMCHRC), Puducherry, India \\ ${ }^{2}$ Department of Microbiology, Mahatma Gandhi Medical College and Research Institute, \\ Puducherry, India \\ *Corresponding
}

\section{A B S T R A C T}

\begin{tabular}{|l|}
\hline Ke y w o r d s \\
Helicobacter pylori, \\
Blood donors, \\
Seropositivity, \\
Puducherry \\
\hline Article Info \\
\hline $\begin{array}{l}\text { Accepted: } \\
\text { 10 January } 2018 \\
\text { Available Online: } \\
\text { 10 February } 2018\end{array}$ \\
\hline
\end{tabular}

Helicobacter pylori induces chronic inflammation of the underlying gastric mucosa causing gastritis and other complications. H. pylori infection once acquired is believed to persist lifelong unless treated. This is a cross-sectional epidemiological study to find the prevalence of the infection in asymptomatic healthy blood donors attending the SVMCHRC blood bank, Puducherry. Analysis of the sera from 99 apparently healthy subjects, based on the serological determination of the $\operatorname{IgM}$ and $\operatorname{IgG}$ antibodies against $H$. pylori, was carried out using a commercially available kit ELISA. There were $59(60 \%)$ males and $40(40 \%)$ females with the age range of 18 to 60 years. Overall seropositivity to Helicobacter pylori among healthy blood donors was $29.2 \% .8(8.08 \%)$ subjects were positive for IgM, 21 (21.2\%) subjects were positive for IgG. Age distribution of $H$. pylori infection showed increasing trend in the young age (21-25 years). A moderate increase in the prevalence of $H$. pylori infection among smokers was noticed. Among Tea/Coffee consumers $25(28.7 \%)$ showed the presence of antibody. Among 26 alcoholics involved in this study only 8 were found to be positive for $\operatorname{IgG}$ and $\operatorname{IgM}$ antibodies. Individuals living with low socioeconomic conditions had high prevalence rates of $H$. pylori.

\section{Introduction}

Helicobacter pylori are bacterial pathogen present in the luminal surface of gastric epithelium.

It induces chronic inflammation of the underlying gastric mucosa causing gastritis and other complications. Prevalence of this disease varies worldwide being low in developed countries and high in developing countries. H. pylori infection once acquired is believed to persist lifelong unless treated (Klein et al., 1994). Most of the infected individual remains asymptomatic. $H$. pylori infection is a co factor in the development of three important upper gastro intestinal diseases: duodenal or gastric ulcer, gastric cancer and gastric mucosa associated lymphoid tissue (MALT) lymphoma (McColl, 2010). Hence, identifying the antibodies in asymptomatic healthy individual by ELISA and treating them is essential. Blood donors are considered as healthy individuals, hence 
their blood samples are examined to determine the seroprevalence of $H$. pylori in population from Puducherry, India.

\section{Materials and Methods}

The study was carried out at the Department of Microbiology, Sri Venkateshwara Medical College Hospital and Research Centre, Pondicherry. A total of 99 healthy individuals between 18-60 years old consecutive donors who were asymptomatic and attended the Sri Venkateshwara Medical College Hospital's Blood Bank, between November 2015 and October 2016 were randomly included. The group comprised of 59 males and 40 females with an age range of 18 to 60 years. Subjects who were previously treated for $H$. pylori infection or who had received antibiotics, proton pump inhibitors or bismuth compounds in the preceding 4 weeks were excluded. Under aseptic precautions, $3 \mathrm{ml}$ blood was collected from blood bag tube (which does not contain any clotting factor) into vacutainer. It was centrifuged at 3000 revolutions per minute for 10 minutes. Serum was separated. The samples were collected in serum tubes and stored in aliquots at $-20^{\circ} \mathrm{C}$ until analysis. All sera were tested for H. pylori IgM and IgG antibodies using ELISA tests (Calbiotech, California, US), according to the standard operating procedures. Ethical clearance was sought and obtained from the SVMCHRC Ethical Committee. Informed written consent was obtained from all subjects before being included in the study. Data was analyzed using Statistical Package for Social Sciences (SPSS), version 21.0.

\section{Results and Discussion}

A total of 99 subjects were included in the study. All the 99 subjects, serum samples were tested for the presence of Helicobacter pylori specific IgG and IgM. 8 (8.08\%) subjects were positive for $\operatorname{IgM}$ i.e. they are currently infected with Helicobacter pylori (Figure 1). $21(21.2 \%)$ subjects were positive for IgG i.e. they had previous $H$. pylori infection and developed antibody. Overall seropositivity to Helicobacter pylori among healthy blood donors was $29.2 \%$. Percentage of $H$. pylori infection was most commonly seen among young adults of age group 21-25 years (Figure 2). Individuals living with low socioeconomic conditions had high prevalence rates of H. pylori (Figure 3). Male preponderance was seen with $59(60 \%)$ males detected to have $H$. pylori antibodies compared to $40(40 \%)$ females (Table 1). Cigarette smoking and Coffee consumption increased the risk of $H$. pylori (Figure 4).

It is difficult for the investigators on $H$. pylori to have sufficient number of adults without gastrointestinal disorders to establish normal prevalence in the healthy population. The only available non-invasive test, the urea breath test is very expensive. Of less invasive tests, serology is most convenient. In this study, the aim was to establish seroprevalence rate among healthy population. Hospital based controls were used, those donating blood, so that blood for ELISA could be drawn when collecting blood without having to subject the individual to venepuncture merely for research purpose.

In this study, there is a significant difference in the prevalence based on gender. This is in concordance with the study results of Kaore et al., (2012) showed higher prevalence in male gender. Age distribution of $H$. pylori infection in this study showed increasing trend in the young age (21-25 years).

This is similar to the observations laid by Tarkhashvili et al., (2009) Shokrzadeh et al., (2012) and Kaore, et al., (2012) which reported increased $H$. pylori infection in age groups of 20-40 years than the older age group. 
Cigarette smoking is an important factor in peptic ulcer disease and dyspeptic symptoms. Since the use of tobacco impairs the immune system (Cope et al., 1992), it was speculated that current cigarette smokers are predisposed to infection. This was examined in a prospective study of patients attending for endoscopy using urease testing. It is known that the link between peptic ulcer and smoking cannot be explained by changes in acid output (Kaufmann D et al., 1990).

In this study a moderate but not significant increase in the prevalence of $H$. pylori infection among smokers was noticed (Figure 4). A limitation of the study is the fact that information on smoking and on alcohol and coffee consumption was ascertained by a selfadministered questionnaire without verification by biological markers.

Self-reports of smoking are typically reasonably accurate (Patrick et al., 1994), but we believe that alcohol consumption, as in other epidemiological studies, has probably been underreported to some extent. Such underreporting could not have produced the observed patterns if alcohol consumption was unrelated to $H$. pylori infection (Brenner et al., 1994).

In this study out of total 99 individuals, 87 consumes tea/coffee, among them 25(28.7\%) showed the presence of antibody (Figure 4). The positive relation between coffee/tea consumption and $H$. pylori infection identified in our study is consistent with results from a cohort study among epidemiologists in which the risk of seroconversion (change from negative to positive results for antibodies to $H$. pylori in serum was 4.6 times higher among those who drank more than 2 cups of caffeinated drinks a day than among the others (Parsonnet et al., 1992). The mechanisms underlying this association require further research.
Among 26 alcoholics involved in this study only 8 were found to be positive for IgG and IgM antibodies (Figure 4). Several studies have reported the relation between alcohol consumption and $H$. pylori infection (Graham et al., 1991; Paunio et al., 1994). Most of them did not find a significant association. A study from Finland found a positive association between alcohol consumption and $H$. pylori infection among younger people (aged 18-35) and a negative association among older age groups (>46) (Paunio et al., 1994). Yet, interpretation and generalization of these findings are difficult because the study was conducted among military staff who had endoscopy for gastric complaints and who drank heavily. The reason for the differences between our results and previous results on the role of alcohol consumption may be how infection was measured. Most previous studies used IgG titres to measure infection (Graham et al., 1991; Paunio et al., 1994).

High IgG titres may persist after elimination of active infection (Meyer et al., 1991), so elimination of infection resulting from alcohol consumption might be missed in studies using IgG titres as markers of infection. Individuals living in countries with low socioeconomic conditions had high prevalence rates of $H$. pylori acquired at an early age (Ally $\mathrm{R}$ et al., 1999). WHO found that the majority of infections occurred in young and middle age groups (25-50 years) more than in other age groups and the factors that predispose the higher colonization rates included poor socioeconomic status and less education in addition to genetic factors. The explanations for the present study are in agreement with approved results of other studies which were mainly due to socioeconomic status and the sample size of the population studied, type of patients, location of the study as well as the mode of transmission whereby spread infection was acquired from person to person or by oral-oral or feco-oral routes. 
Fig.1 Prevalence of seropositivity to $H$. pylori antibodies by different antibody class

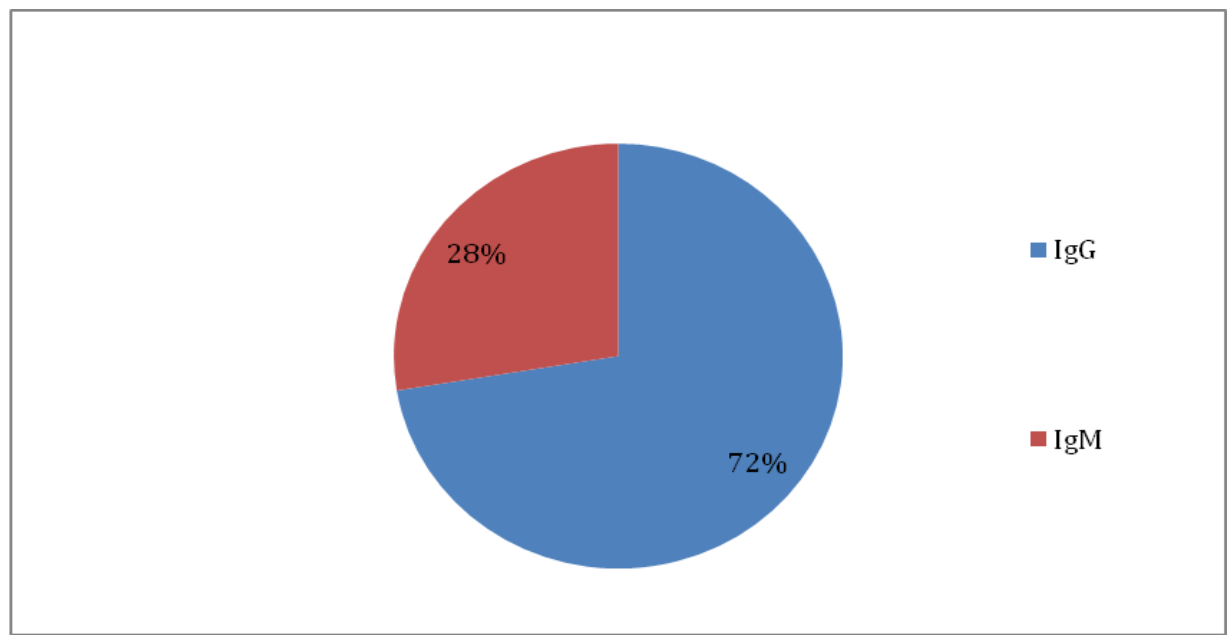

Fig.2 Frequency of detection of antibody classes of $H$. pylori infection among adult blood donors according to age

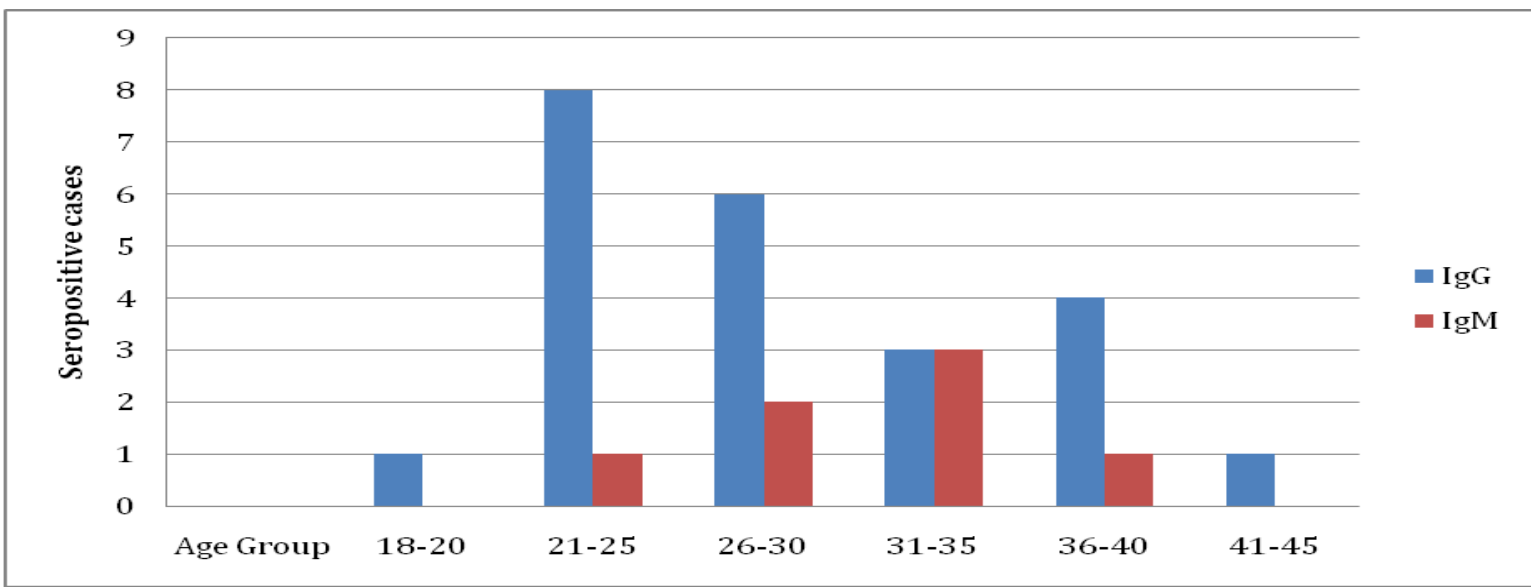

Fig.3 Frequency of detection of antibody classes of $H$. pylori infection among adult blood donors based on Socio economic class

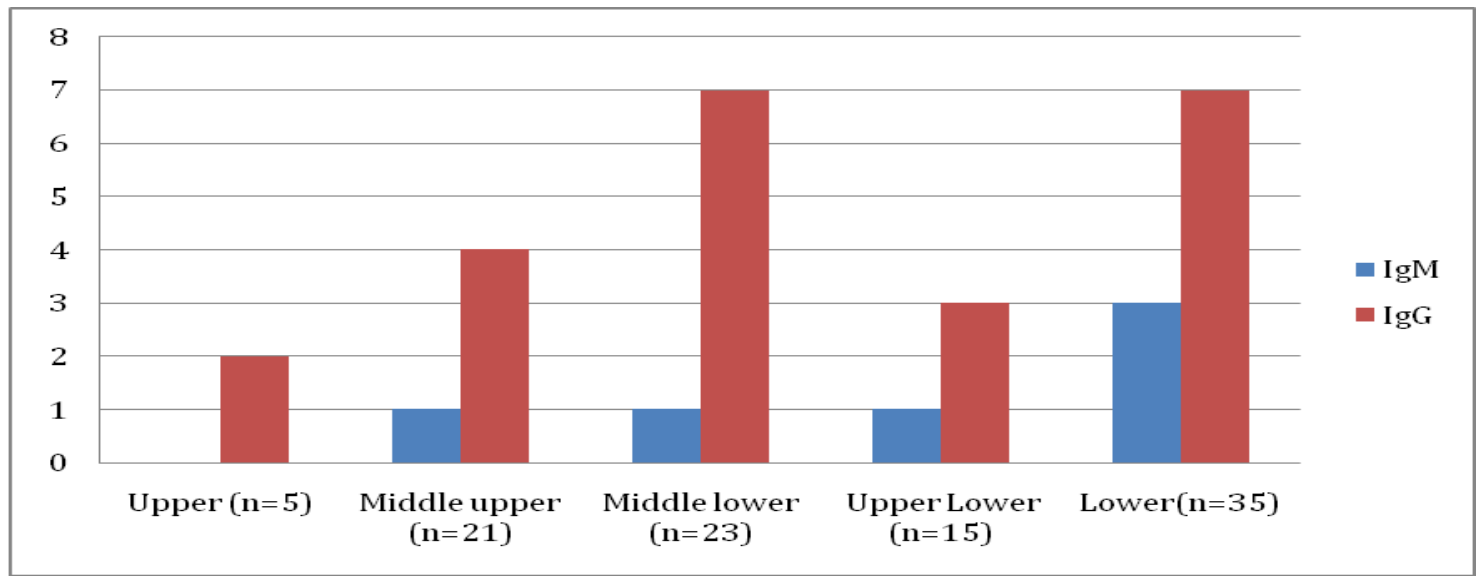


Fig.4 Lifestyle factors influencing $H$. pylori infection

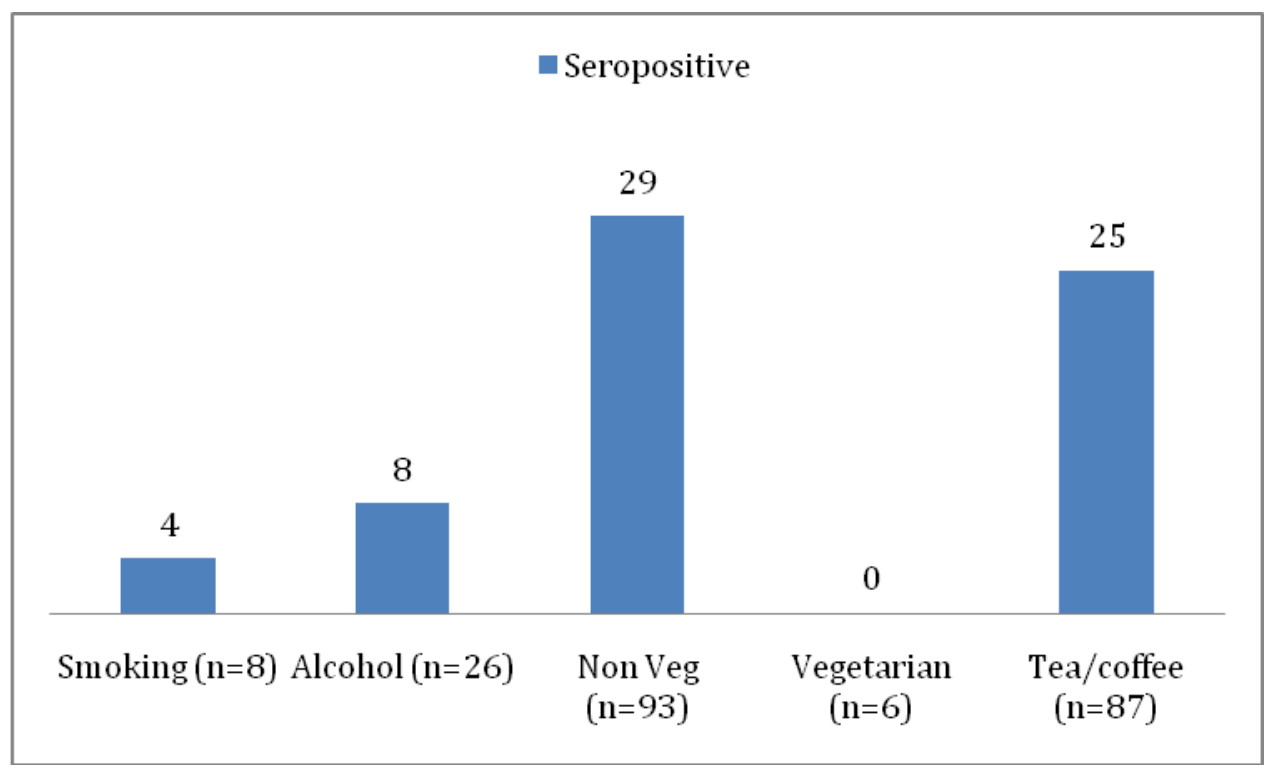

Table.1 H. pylori Infection distribution based on gender

\begin{tabular}{|c|c|c|c|c|}
\hline Gender & \multicolumn{2}{|c|}{ IgG } & \multicolumn{2}{|c|}{ IgM } \\
\hline & Number & Percentage & Number & Percentage \\
\hline Male $(n=59)$ & 14 & $23.7 \%$ & 4 & $6.77 \%$ \\
\hline Female $(n=40)$ & 7 & $17.5 \%$ & 4 & $6.77 \%$ \\
\hline
\end{tabular}

The present study has a major limitation that association of $H$. pylori infection with lifestyle related modifiable factors was not accessed. There is a need of another broader study in this region, assessing the association of different demographic and life-style factors and pre-existing conditions like diabetes mellitus with prevalence of $H$. pylori infection and follow-up of the patients after treatment and life-style modifications.

Although $H$. pylori infection is commonly acquired during childhood, active infection may be acquired and eliminated throughout adulthood. This study of patients in a general practice found no significant relation between smoking and active $H$. pylori infection. Drinking alcohol seemed to protect against active $H$. pylori infection. This protective effect was dose dependent. Drinking coffee was associated with an increased prevalence of active $H$. pylori infection. The protective effect of alcohol on active $H$. pylori infection may be related to its antimicrobial effects.

A set of serological tests may give more accurate determinations of $H$. pylori infection than one test detecting specific antibody or bacterial antigen. In this study, only one serological test was performed, which showed H. pylori seropositivity was $21 \%$ by ELISA $\mathrm{IgG}$ antibodies.

When the infection was contracted is important, especially because $H$. pylori is known as a life-long infection and it colonizes the human stomach for a long period of time until it causes severe infections; thus, individuals might been infected recently and more severe complications have yet to be produced. Though, the prevalence of $H$. pylori gastritis and associated abdominal symptoms 
are high in number, serious gastrointestinal complications develop in few. Absolute prevention of these complications and relief from the distressing abdominal symptoms can be achieved through early detection by conventional and affordable diagnostic methods and empirical treatment with anti $H$. pylori therapy.

\section{Acknowledgement}

The authors express their appreciation to the blood donors and staff of laboratory of SVMCHRC who participated in this study. The authors also wish to thank Dr. Purushothama Rajkumar, Department of Ophthalmology, Aravind eye hospital, Puducherry.

\section{References}

Ally R, Mitchell H, Segal H. Cag A positive $H$. pylori a plenty in South Africa: the first systemic study of $H$. pylori infection in systemic children in Soweto. Gut 1999; 45: 97-98

Brenner H, Loomis D. Varied forms of bias due to non-differential error in measuring exposure. Epidemiology 1994; 5: 510-7.

Cope, G.F. \&Heatley, R.V. Cigarette smoking and intestinal defences. Gut 1992, 33: 721-3.

Graham DY, Malaty HM, Evans DG, Evans DJ, Klein PD, Adam E. Epidemiology of Helicobacter pylori in an asymptomatic population in the United States. Effect of age, race and socioeconomic status. Gastroenterology. 1991; 100:1495-501.

Kaore NM, Nagdeo NV, Thombare VR. Comparative evaluation of the diagnostic tests for Helicobacter pylori and dietary influence for its acquisition in dyspeptic patients: A rural hospital based study in central India. JCDR 2012; 6:636-41.

Kaufmann, D., Wilder-Smith, C.H., Kempf, M. et al., Cigarette smoking, gastric acidity and peptic ulceration. What are the relationships? Dig Dis Sci 1990, 35: 1482-87.

Klein PD, Gilman RH, Barua RL, Diar F, Smith EO, Graham DY. The epidemiology of Helicobacter pylori in Peruvian children between 6 and 30 months of age. Am J Gastroenterol 1994; 89: 2196-200.

McColl KE. Helicobacter pylori infection. N Engl Med 2010; 362(17):1597-1604.

Meyer B, Werth B, Beglinger C, Dill S, Drewe J, Vischer WA, et al., Helicobacter pylori infection in healthy people: a dynamic process? Gut 1991; 32:347-50.

Parsonnet J, Blaser MJ, Perez PG, Hergrett BN, Tauxe RV. Symptoms and risk factors of Helicobacter pylori infection in a cohort of epidemiologists. Gastroenterology1992; 102:41-6.

Patrick DL, Cheadle A, Thompson DC, Diehr P, Koepsell T, Kinne S. The validity of self-reported smoking: a review and meta-analysis. Am J Public Health1994; 84:1086-93.

Paunio M, Höök-NJ, Kosunen TU, Vainio U, Salaspuro M, Mäkinen J, et al., Association of alcohol consumption and Helicobacter pylori infection in young adulthood and early middle age among patients with gastric complaints. Eur J Epidemiol.1994; 10:205-9.

Shokrzadeh L, Baghaei K, Yamaoka Y, Shiota S, Mirsattari D, Porhoseingholi A, et al., Prevalence of Helicobacter pylori infection in dyspeptic patients in Iran. Gastroenterol Insights 2012; 4:24-7.

Tarkhashvili N, Beriashvili R, Chakvetadze N, Moistsrapishvili M, Chokheli M, Sikharulidze M, et al., Helicobacter pylori infection in patients undergoing upper endoscopy, Republic of Georgia. Emerg Infect Dis 2009; 15:504-5.

\section{How to cite this article:}

Ramya Priyadarshini, D., Joshy M. Easow and Vinod, R. 2018. Seroprevalence of Helicobacter Pylori among Healthy Blood Donors. Int.J.Curr.Microbiol.App.Sci. 7(02): 817-822.

doi: https://doi.org/10.20546/ijcmas.2018.702.103 